\title{
Diagnostic difficulty in polycystic ovary syndrome due to an LH- $\beta$-subunit variant
}

H Kurioka, K Takahashi, M Irikoma, M Okada, T Ozaki, T Ueda and K Miyazaki

Department of Obstetrics and Gynecology, Shimane Medical University, Izumo 693-8501, Japan

(Correspondence should be addressed to H Kurioka, Department of Obstetrics and Gynecology, Shimane Medical University, 89-1 Enya-cho, Izumo 693-8501, Japan)

\begin{abstract}
We initially failed to confirm a case of polycystic ovary syndrome (PCOS) because underestimation of LH concentrations due to a variant form of this hormone resulted in a misleadingly low LH/FSH ratio. A 26-year-old woman presented to our hospital with infertility. Given the presence of bilateral polycystic ovaries, oligomenorrhea and hirsutism, PCOS was suspected, but a normal LH/FSH ratio as measured by RIA led to diagnostic problems. When we remeasured LH and FSH using a chemical luminescence enzyme immunoassay (CLEIA), the ratio of the LH concentration measured by RIA to that measured by CLEIA was 0.29 , and the ratio of LH to FSH measured by CLEIA was 3.3 compared with 0.81 measured by RIA. We then diagnosed PCOS. The point mutations $\operatorname{Trp}^{8}$ to $\mathrm{Arg}^{8}$ and Ile ${ }^{15}$ to $\mathrm{Thr}^{15}$ in the LH subunit were detected in the corresponding gene. The patient's LH status represented variant and wild-type LH equally. She was therefore diagnosed as heterozygous for the mutant LH- $\beta$. Histologic assessment of ovarian tissue after laparoscopic biopsy was compatible with a polycystic ovary.
\end{abstract}

European Journal of Endocrinology 140 235-238

\section{Introduction}

Luteinizing hormone (LH) is a member of the glycoprotein hormone family that includes human chorionic gonadotropin, follicle-stimulating hormone (FSH), and thyroid-stimulating hormone. These hormones possess $\alpha$ and $\beta$ subunits; the $\beta$ subunit confers the hormonal activity. Both subunits are glycosylated at specific residues, and the degree of glycosylation modulates the biological activity of LH. A certain degree of microheterogeneity occurs in glycosylation, contributing to variability in bioactivity and biochemical properties.

Recently developed ultrasensitive immunoassays (1, 2) using monoclonal antibodies for the measurement of LH have resulted in a new risk of error. These monoclonal antibodies may be highly specific to indigenous LH; some studies have noted restricted reactivity of the monoclonal antibodies in certain populations $(3,4)$. In previous studies $(5,6)$ PCR was used to specifically amplify the LH- $\beta$ gene. Subsequent nucleotide sequencing revealed two point mutations in the gene coding for the LH- $\beta$ subunit, resulting in two amino acid replacements: $\operatorname{Trp}^{8}$ (TCG) was changed to $\operatorname{Arg}(\mathrm{CGG})$ and Ile $^{15}$ (ATC) to Thr(ACC). Further examination by restriction fragment length polymorphism revealed the existence of homozygotes and heterozygotes for the mutant LH- $\beta$ gene among family members, indicating a genetic basis for an abnormal LH molecule. Although the homozygotes varied in degree of clinical ovarian dysfunction, the direct effects of the mutant LH on gonadal dysfunction remained unclear. Several commercially available LH assays using $\alpha / \beta$ heterodimerspecific monoclonal antibodies fail to detect variant forms of LH, or detect them weakly, potentially leading to errors in LH measurements.

We report here on a patient with polycystic ovary syndrome (PCOS) in whom serum LH concentrations and the LH/FSH ratio appeared to be normal when measured with a conventional immunoassay kit.

\section{Case report}

The patient was a 26-year-old nulligravida who had been married for 2 years. She presented to our clinic on 13 July 1995, complaining of infertility. Menarche had occurred at age 13, but oligomenorrhea had been noted. Her history included acute pancreatitis at age 23 . Her stature was normal and she was not obese (body mass index: $21.5 \mathrm{~kg} / \mathrm{m}^{2}$ ). Hirsutism was present and her Ferriman score (7) was 10. Pelvic examination was normal, but transvaginal ultrasonography revealed bilaterally polycystic ovaries. From these clinical 
Table 1 Hormonal examinations in the early follicular phase.

\begin{tabular}{lll}
\hline Date & \multicolumn{1}{c}{ Substance (method) } & \multicolumn{1}{c}{ Value } \\
\hline 16 August '95 & LH (SPAC-S LH) & $4.0 \mathrm{mlU} / \mathrm{ml}$ \\
& FSH (SPAC-S FSH) & $7.6 \mathrm{mlU} / \mathrm{ml}$ \\
& PRL (SPAC-S PRL) & $5.3 \mathrm{ng} / \mathrm{ml}$ \\
& $\mathrm{E}_{2}$ (coat-a-count $\mathrm{E}_{2}$ ) & $28 \mathrm{pg} / \mathrm{ml}$ \\
& T (total testosterone kit) & $33.6 \mathrm{ng} / \mathrm{dl}$ \\
25 February '96 & $\mathrm{E}_{1}$ (estrone test set) & $99 \mathrm{pg} / \mathrm{ml}$ \\
& $\mathrm{E}_{2}$ (coat-a-count $\mathrm{E}_{2}$ ) & $45 \mathrm{pg} / \mathrm{ml}$ \\
& T (total testosterone kit) & $20.1 \mathrm{ng} / \mathrm{dl}$ \\
& ASD (coat-a-count ASD) & $2.4 \mathrm{ng} / \mathrm{ml}$ \\
& DHEA-S (coat-a-count DHEA-S) & $1830 \mathrm{ng} / \mathrm{ml}$
\end{tabular}

$\mathrm{E}_{2}$, estradiol; $\mathrm{E}_{1}$, estrone; $\mathrm{T}$, testosterone; $\mathrm{ASD}$, androstenedione; DHEA-S, dehydroepiandrosterone-sulfate.

features, PCOS was strongly suspected. The results of hormonal determinations by RIA in the early follicular phase (Table 1) indicated a normal LH/FSH ratio, which by Japanese diagnostic criteria (8) was not compatible with PCOS. We re-evaluated the hormonal concentrations using a chemical luminescence enzyme immunoassay (CLEIA; IMMULYZE, Nippon Diagnostic Products Corporation, Tiba, Japan). Comparison of the results obtained by the two methods showed a broad disparity in LH concentrations which by RIA were 6.7 $\mathrm{mIU} / \mathrm{ml}$ and by CLEIA were $23.0 \mathrm{mIU} / \mathrm{ml}$. The FSH concentrations obtained by the two methods showed almost the same results (by RIA $8.3 \mathrm{mIU} / \mathrm{ml}$ and by CLEIA $6.9 \mathrm{mIU} / \mathrm{ml}$ ). An LH-releasing hormone (LHRH) test (100 $\mu \mathrm{g}$ LHRH injected i.v.) showed a high response of LH measured by CLEIA (Fig. 1), leading to diagnosis of PCOS and variant LH- $\beta$. The ratio of the LH concentration found by RIA to that found by CLEIA was 0.29 , suggesting that the patient was heterozygous for the mutant LH- $\beta$ (9).

DNA analysis using PCR with direct sequencing by a method previously described (9) was employed. Genomic DNA was isolated from peripheral blood lymphocytes using Sepa Gene nucleic acid isolation reagents

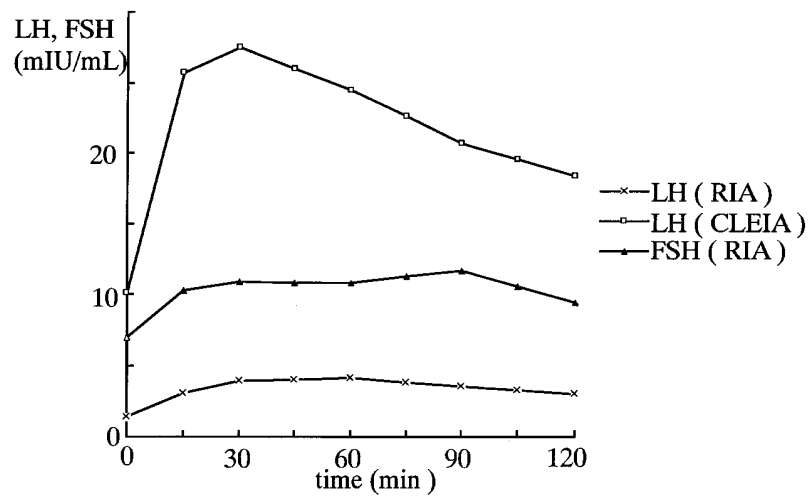

Figure $1 \mathrm{An}$ LHRH test (100 $\mu \mathrm{g}$ LHRH injected i.v.) showed an increased response of $\mathrm{LH}$ as measured by CLEIA.
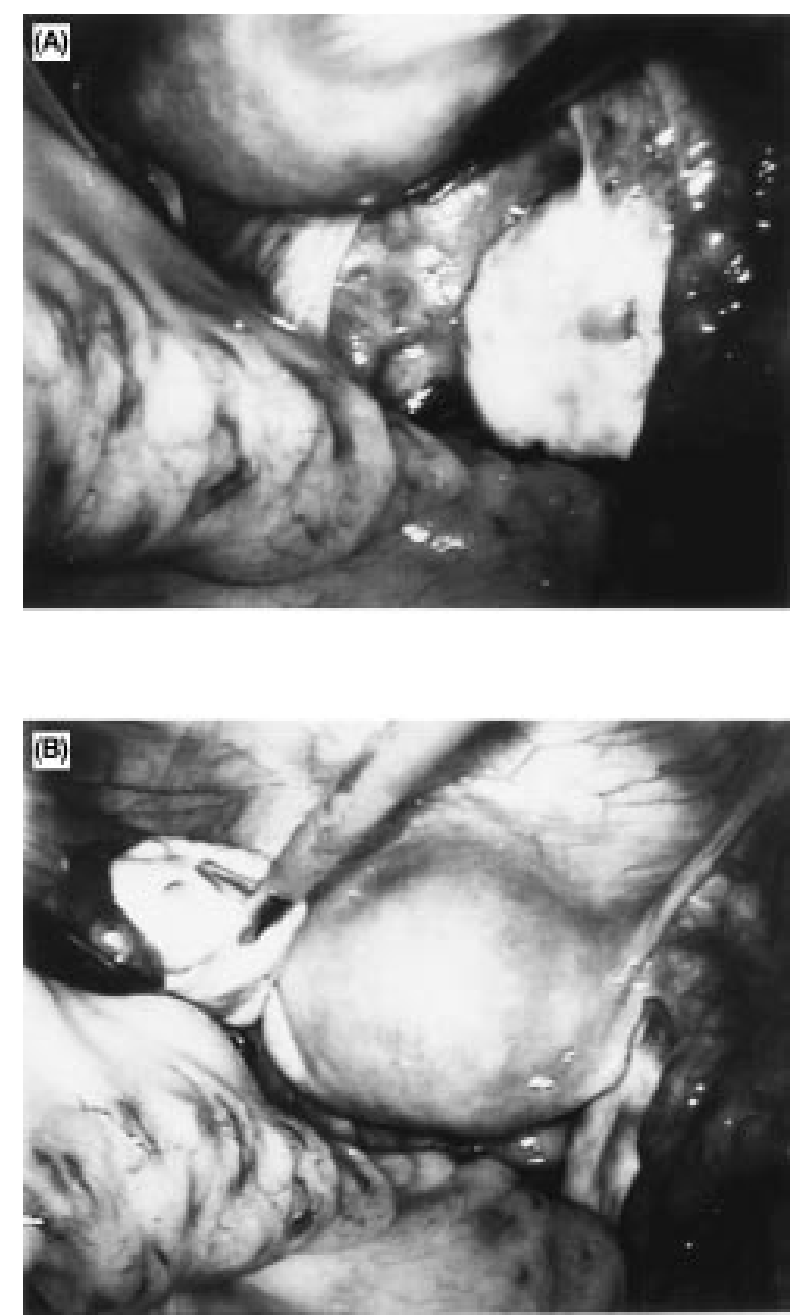

Figure 2 Laparoscopic appearance of enlarged polycystic ovaries. (A) right; (B) left.

(Sanko Junyaku, Tokyo, Japan). The gene encoding the LH- $\beta$ subunit was sequenced, and two base changes in the N-terminal region were identified. The first mutation (codon 8, TCG to CGG) changes tryptophan to arginine, and the second (codon 15, ATC to ACC) changes isoleucine to threonine. The patient's LH status represented variant and wild-type LH equally. No other infertility factors were recognized. She was treated with clomiphene citrate (Clomid; Shionogi Seiyaku, Osaka, Japan) for two courses of $100 \mathrm{mg} /$ day for 5 days and two courses of $150 \mathrm{mg} /$ day for 5 days without successful ovulation. Two cycles of controlled ovarian hyperstimulation using pure FSH (FERTINORM P; Serono Japan, Tokyo, Japan) were attempted, but she did not conceive and developed mild ovarian hyperstimulation syndrome which was treated in the outpatient clinic. Laparoscopy on 12 August 1997 showed polycystic ovaries measuring $4 \times 3.5 \times 3.0 \mathrm{~cm}$ on both sides (Fig. 2). The histological appearance of a biopsy specimen was 
compatible with PCOS. She underwent laparoscopic ovarian electrocautery.

\section{Discussion}

Controversy surrounds the diagnosis of PCOS, with different authors using differing criteria to define the syndrome. In the UK, polycystic ovaries detected on an ultrasonogram are generally accepted as the unifying diagnostic criterion. Additional biological abnormalities (elevated serum concentrations of LH, androgens, and/ or insulin) may occur together with the characteristic sonographic appearance (10). However, the diagnosis of PCOS was originally based on clinical symptoms and biochemical parameters. Some investigators use a single biochemical marker such as elevated serum LH concentration (11) or high androgen levels (10) to define PCOS. Recent studies claim that there is a tendency to overestimate PCOS in the USA (12). Japanese diagnostic criteria for PCOS (8) use a combination of ultrasound, clinical symptoms and an LH/FSH ratio $>1$.0. LH concentrations are an important consideration in diagnosing PCOS. An LH variant with two point mutations in the $\beta$-subunit gene was recently reported in Japan $(5,13)$.

In the present case, LH immunoassays using various antibodies gave different results. Ultrasensitive immunoassays for LH $(1,2)$, based on the sandwich technique and the use of monoclonal antibodies, have revealed some interesting aspects. The risk that monoclonal antibodies may be too specific has been pointed out (4), especially in cases where the protein or glycoprotein, like LH, is clearly microheterogeneous. With two anti-LH monoclonal antibodies directed to epitopes, only intact dimers were found which had restricted reactivity with $\mathrm{LH}$ at various rates (14) in a randomly selected population. The carrier frequency of the variant $L H-\beta$ allele varied from $7.1 \%$ in USA Hispanics to $41.9 \%$ in the Lapps of Northern Finland (15). However, about $10 \%$ of the population of Japan shows dissociation of $\mathrm{LH}$ values. The variant $\mathrm{LH}-\beta$ allele tended to be more common in populations from Nothern Europe as compared with those from Asia. Underestimation of $\mathrm{LH}$ concentrations in a serum sample can impair detection of the ovulatory LH peak or artefactually decrease the LH/FSH ratio, leading to failure to diagnose PCOS. Patients homozygous or heterozygous for the LH- $\beta$ gene mutation were discovered by PCR and restriction fragment length polymorphism, an expensive approach not suitable for routine examination.

Nilsson et al. (14) performed two immunofluorimetric assays using monoclonal antibodies: assay 1 recognized only wild-type LH, while assay 2 recognized variant and wild-type LH equally. In assay 1 , the capture monoclonal antibody recognized a conformational epitope present in the intact $\alpha / \beta-\mathrm{LH}$ dimer but not in the subunits, and the detection monoclonal antibody recognized the $\alpha$ subunit. Assay 2 used two LH- $\beta$ subunit-specific monoclonal antibodies. The ratio of results from assay 1 to those from assay 2 indicated the LH status: wild-type $>0.9$, heterozygous $0.2-0.9$, and homozygous <0.15. Similarly, we established (9) a screening method for this genetic variant of LH, measuring hormonal concentrations by IMMULYZE (9), a random access instrument using recently developed CLEIA techonology. IMMULYZE LH is a solid-phase test which uses an alkaline phosphatase-conjugated polyclonal antibody as a tracer and polystyrene beads coated with monoclonal antibodies specific for LH. LH values measured by IMMULYZE LH ordinarily show a good correlation with SPAC-S LH, which uses RIA. SPAC-S LH, purchased from Daiichi Radioisotope Laboratory (Tokyo, Japan), uses an immunoradiometric assay method (1) and two different monoclonal antibodies, which react with the $\beta$ subunit and intact dimer respectively. The relationship is linear: $y=0.982 x+1.396, r=0.970$, where $y$ is the $\mathrm{LH}$ value found by IMMULYZE LH, and $x$ is the LH value found by SPAC S-LH. Furui et al. (5) have shown that measurement by SPAC-S LH is affected by point mutations of the LH- $\beta$ gene. Therefore, we believe that the difference between tests might be due to poor recognition of LH in such patients by intact LH dimer monoclonal antibodies. Adding serum from SPAC-S LH, including LH coated with anti-LH $\beta$ monoclonal antibodies, to the IMMULYZE LH preparation resulted in decreased LH values.

From these results, we concluded that our patient represented an immunologically anomalous LH variant. We have reported (9) that a ratio of SPAC-S LH to IMMULYSE LH of less than 0.5 or equal to 0.5 is abnormal, permitting recognition of PCOS occurring together with a point mutation of the LH- $\beta$ gene. When a patient with bilateral polycystic ovaries by ultrasound, oligomenorrhea and hirsutism shows a normal LH/FSH ratio, the presence of a genetic LH variant should be suspected. Rajkhowa et al. (15) have reported that occurrence of the variant was not generally increased in women with PCOS and the presence of the variant did not alter the clinical or hormonal expression of the disorder in women with PCOS, but was over-represented among obese women with PCOS. Variant LH without PCOS has been associated with elevated total serum testosterone (16). The biological attributes of variant and wild-type LH appear to differ by in vitro bioassay and by circulatory half-time measurements (16). The in vitro bioactivity of the LH variant was significantly increased. The higher bioactivity but faster elimination of the variant LH could change the kinetics of its action in vivo. Connections between the mutation LH- $\beta$ and infertility are not well known. The LH variant may contribute to some diseases affecting the pituitarygonadal axis, although atypical clinical features were not seen in the present case of PCOS coexisting with the LH variant. 


\section{References}

1 Odell WD \& Griffin J. Two-monoclonal-antibody 'sandwich'-type assays of human lutropin, with no cross reaction with choriogonadotropin. Clinical Chemistry 198733 1603-1607.

2 Haavisto AM, Dunkel L, Pettersson K \& Huhtaniemi I. LH measurements by in vitro bioassay and a highly sensitive immunofluorometric assay improve the distinction between boys with constitutional delay of puberty and hypogonadotropic hypogonadism. Pediatric Research 199027 211-214.

3 Pettersson KS \& Soderholm JR-M. Individual differences in lutropin immunoreactivity revealed by monoclonal antibodies. Clinical Chemistry 199137 333-340.

4 Pettersson KS, Ding Y-Q \& Huhtaniemi I. An immunologically anomalous luteinising hormone variant in a healthy woman. Journal of Clinical Endocrinology and Metabolism 1992 74 164-171.

5 Furui K, Suganuma N, Tsukahara S, Asada Y, Kikkawa F, Tanaka M, Ozawa T \& Tomoda Y. Identification of two point mutations in the gene coding luteinizing hormone (LH) $\beta$-subunit, associated with immunologically anomalous LH variants. Journal of Clinical Endocrinology and Metabolism 199478 107-113.

6 Suganuma N, Furui K, Furuhshi M, Asada Y, Kikkwa F \& Tomoda Y. Screening of the mutation in luteinizing hormone $\beta$-subunit in patients with menstrual disorders. Fertility and Sterility 199563 989-995.

7 Ferriman D \& Gallwey JD. Clinical assessment of body hair growth in women. Journal of Clinical Endocrinology and Metabolism 1961 21 1440-1447.

8 The Committee for Reproductive and Endocrine in Japan: Society of Obstetrics and Gynecology. Annual report (1991-1992) for the determination of diagnostic criteria for polycystic ovary syndrome (Japanese). Acta Obstetrica et Gynaecologica Japonica 199345 1359-1367.

9 Karino K, Kunishi H, Masuda J, Ozaki T \& Takahashi K. Different results obtained by IMMULYZE and RIA method in measurement of serum LH and estradiol levels. Igaku Kensa 199746 (Suppl) 673 .
10 Balen AH, Conway GS, Kaltsas G, Techatraisak K, Manning PJ, West C \& Jacobs HS. Polycystic ovary syndrome: the spectrum of disorder in 1741 patients. Human Reproduction $1995102107-$ 2111.

11 Scheele F, Hompes PGA, van der Meer M, Schoute E \& Schoemaker J. The effects of gonadotropin-releasing hormone agonist on treatment with low-dose follicle stimulating hormone in polycystic ovary syndrome. Human Reproduction 19935 699704.

12 Abdel GA, Khatim MS, Mowafi RS, Alnaser HM, Muharib NS \& Shaw RW. Implications of ultrasonically diagnosed polycystic ovaries. II. Studies of dynamic and pulsatile hormonal patterns. Human Reproduction 19927 458-461.

13 Okuda K, Yamada T, Imoto H, Komatsubara H \& Sugimoto O. Antigenic alternations of an anomalous human luteinizing hormone caused by two chorionic gonadotropin-type aminoacid substitutions. Biochemical and Biophysical Resarch Communications $1994200584-590$.

14 Nilsson C, Petterson K, Millar RP, Coerver KA, Matzuk MM \& Huhtaniemi IT. Worldwide frequency of a common genetic variant of luteinizing hormone: an international collaborative research. Fertility and Sterility 199767 998-1004.

15 Rajkhowa M, Talbot JA, Jones PW, Pettersson K, Haavisto AM, Huhtaniemi I \& Clayton RN. Prevalence of immunological LH $\beta$ subunit variant in a UK population of healthy women and women with polycystic ovary syndrome. Clinical Endocrinology 199543 297-303.

16 Haavisto AM, Petterson K, Bergendahl M, Virkamaki A \& Huhtaniemi I. Occurrence and biological properties of a common genetic variant of luteinizing hormone. Journal of Clinical Endocrinology and Metabolism 199580 1257-1263.

Received 5 May 1998

Accepted 27 November 1998 